\title{
Overexpression of circACTR2 in Gestational Diabetes Mellitus Predicts Intrauterine Death, Fetal Malformation, and Intrauterine Infection
}

\author{
Can Zhu' \\ Yuning Liu ${ }^{2}$ \\ Haiying $\mathrm{Wu}^{\prime}$ \\ 'Department of Obstetrics and \\ Gynecology, Henan Provincial People's \\ Hospital, Zhengzhou City, Henan \\ Province, 450003, People's Republic of \\ China; ${ }^{2}$ Department of Neonatal \\ Intensive Care Unit, Henan Provincial \\ People's Hospital, Zhengzhou City, \\ Henan Province, 450003, People's \\ Republic of China
}

Background: CircRNA actin-related protein 2 homolog (circACTR2) has been reported to participate in high glucose-induced disorders, while its role in gestational diabetes mellitus (GDM) is unknown. This study analyzed the expression pattern of circACTR2 in GDM and evaluated its predictive value for GDM and its adverse events.

Methods: ClircACTR2 expression in plasma of 200 pregnant females with a gestational age of about 1 month was analyzed once per month using RT-qPCR. The development of GDM was monitored until delivery. Adverse events, including premature delivery, miscarriage, intrauterine distress, intrauterine death, fetal malformation, intrauterine infection, hypertension, and macrosomia, were recorded.

Results: During the follow-up, a total of 70 patients were diagnosed with GDM. The 70 GDM patients showed significantly higher plasma circACTR2 levels compared to the remaining 130 pregnant females. With the median plasma circACTR2 level in the first month as the cutoff value, the 200 patients were divided into the high and low circACTR2 level groups, and their GDM-free curves were plotted and compared. Patients in high circACTR2 level group showed a higher incidence of GDM. Moreover, among the 70 patients diagnosed with GDM, high circACTR2 levels were also closely correlated with higher rates of premature delivery, miscarriage, intrauterine death, fetal malformation, intrauterine infection, and hypertension, but not with macrosomia and intrauterine distress.

Conclusion: CircACTR2 is overexpressed in GDM. The increased plasma circACTR2 levels in pregnant women predict GDM, and higher plasma circACTR2 levels in GDM patients predict multiple adverse events.

Keywords: gestational diabetes mellitus, circACTR2, adverse events

\section{Introduction}

Gestational diabetes mellitus (GDM) is glucose intolerance during pregnancy. ${ }^{1}$ GDM is usually caused by reduced insulin secretion during late pregnancy. ${ }^{2}$ About $14 \%$ of pregnant women develop GDM during pregnancy worldwide. ${ }^{3,4}$ The occurrence of GDM affects pregnancy and delivery and contributes to the occurrence of type 2 diabetes, heart diseases, metabolic problems, and pediatric cardiovascular diseases in both pregnancy and late-life. ${ }^{5,6}$ GDM is usually treated with insulin injection. ${ }^{7}$ However, currently, available treatment approaches cannot completely prevent pregnant women and infants from developing complications. Therefore, early diagnosis and prevention are still critical.
Correspondence: Haiying Wu Department of Obstetrics and Gynecology, Henan Provincial People's Hospital, No. 7 Weiwu Road, Jinshui District, Zhengzhou City, Henan

Province, 450003, People's Republic of China

Email haiyingwuhenan@163.com 
Previous studies on the molecular pathogenesis of GDM have revealed the involvement of multiple molecular alterations in GDM. ${ }^{8}$ For instance, differences in circular RNAs (circRNAs) expression profile in placentas have shown diagnostic potentials for GDM., ${ }^{9,10}$ CircRNAs are covalently closed RNA transcripts with no or limited capacity for protein-coding, but they participate in human diseases mainly by regulating related protein synthesis. ${ }^{11}$ Although a considerable number of differentially expressed circRNAs with critical functions in GDM have been identified, ${ }^{11}$ the clinical values of circRNAs for GDM treatment and prediction and their roles in GDM development and progression remain hardly known. Circular actin-related protein 2 homolog (circACTR2) is a novel circRNA that participates in high glucose-induced disorders, ${ }^{12}$ which contribute to GDM development. We speculated that circACTR2 might participate in GDM. Therefore, this study was carried out to analyze the role of circACTR2 in GDM.

\section{Materials and Methods}

\section{Participants and Follow-Up}

This study enrolled a total of 200 pregnant women with a gestational age of $1.02 \pm 0.23$ months at Henan Provincial People's Hospital between March 2019 and January 2020. During this period, more than 3000 pregnant women were admitted to our hospital. Among them, 1000 were willing to participate. We randomly selected 200 to participate. Ethics approval was obtained from our hospital (No. 2019-10). All 200 participants did not have previous and family history of diabetes and were followed up weekly for a total of 30 weeks to monitor the occurrence of GDM. GDM was diagnosed based on the following criteria: 1) plasma glucose level higher than $180 \mathrm{mg} / \mathrm{dl}$ at $1 \mathrm{~h}$ post glucose load, 2) plasma glucose level higher than $153 \mathrm{mg} / \mathrm{dl}$ at $2 \mathrm{~h}$ post glucose load, and 3) plasma glucose under fasting condition higher than $92 \mathrm{mg} / \mathrm{dl}$. Besides that, adverse events, including premature delivery, miscarriage, intrauterine death, fetal malformation, intrauterine infection, hypertension, macrosomia, and intrauterine distress, were monitored until delivery. All participants provided written informed consent.

\section{Preparation of Plasma Samples}

On the day after admission, all participants fasted overnight, and blood $(5 \mathrm{~mL})$ was extracted from the elbow vein of each participant. Blood samples were immediately transferred to EDTA tubes and centrifuged at 2000x g for $15 \mathrm{~min}$ at $4^{\circ} \mathrm{C}$ to remove the blood cells. A sample of about $2.1 \mathrm{~mL}$ plasma was yielded from $5 \mathrm{~mL}$ blood sample. All plasma samples were divided into two parts. One part was immediately subjected to RNA preparation, and the other part was stored in liquid nitrogen.

\section{Preparation of RNA Samples}

Total circulating RNAs were isolated from plasma samples using Direct-zol RNA Purification Kit (Zymo Research) in strict accordance with the manufacturer's instructions. Prior to further analysis, genomic DNA was removed from all RNA samples by incubating with DNase I for $90 \mathrm{~min}$ at $37^{\circ} \mathrm{C}$. RNA concentration and integrity were analyzed using Agilent 2100 Bioanalyzer system. All RNA samples had concentrations higher than $200 \mathrm{ng} / \mu \mathrm{L}$ and RIN values higher than 8. Otherwise, RNA isolation and genomic DNA removal were repeated.

\section{RT-qPCR Assay}

Quantification of circACTR2 in all RNA samples from plasma samples was performed through reverse transcriptions (RTs). All RTs were performed with SSRT III system (Thermo Fisher Scientific) using 1500 ng RNA as a template at conditions of $5 \mathrm{~min}$ at $25^{\circ} \mathrm{C}, 20 \mathrm{~min}$ at 55 ${ }^{\circ} \mathrm{C}$, and $10 \mathrm{~min}$ at $85^{\circ} \mathrm{C}$. With GAPDH as an internal control, qPCRs were performed to analyze circACTR2 levels in all samples. All qPCR mixtures were prepared using SYBR Green Master Mix (Bio-Rad) with $1 \mu \mathrm{L}$ cDNA sample as template. All qPCRs were performed on Applied Biosystems ${ }^{\mathrm{TM}}$ StepOne ${ }^{\mathrm{TM}}$ Real-Time PCR System at cycling conditions of $2 \mathrm{~min}$ at $95^{\circ} \mathrm{C}$ and 40 cycles of $95^{\circ} \mathrm{C}$ for $10 \mathrm{~s}$ and $58^{\circ} \mathrm{C}$ for $10 \mathrm{~s}$. Primer sequences used in qPCRs were circACTR2 forward 5'TGTGCTTTCTGGAGGGTCTACT-3' and reverse 5'TGCCTCATCACCAACCATA AG-3' and GAPDH forward 5'-CTGGGCTACACTGAGCACC-3' and reverse 5'AA GTGGTCGTTGAGGGCAATG-3'. Ct values from all reactions were analyzed using the $2^{-\Delta \Delta \mathrm{Ct}}$ method.

\section{Statistical Analysis}

All data were expressed as average values of three technical replicates and were used for data comparisons and analyses. Comparisons of the two independent groups were performed with unpaired $t$-test. With the median plasma circACTR2 level of the 200 participants at admission as the cutoff value, the 200 participants were divided into high and low circACTR2 level groups. The follow-up data were used to 
plot GDM-free curves for both groups. The comparison of GDM-free free curves was performed using Log rank test. With the median plasma circACTR2 level of the 70 GDM patients at admission as the cutoff value, the 70 GDM patients were further divided into high and low circACTR2 level groups. The correlation between adverse events and plasma circACTR2 levels in these patients was analyzed by Chi-squared test or Fisher's exact test. A $p<0.05$ was considered statistically significant.

\section{Results}

\section{Analysis of the Baseline Data of the 200 Participants}

In this study, the 200 participants were at $27.8 \pm 3.8$ years on average. Among them, 150 participants were younger than 30 years, and 50 were older than 30 years. The prepregnancy BMI was within the normal range in all cases, with a mean value of $21.4 \pm 3.1$. Fasting blood glucose in all cases was within the normal range with a mean value of $4.71 \pm 0.29 \mathrm{mmol} / \mathrm{L}$. Among the 200 participants, miscarriage and intrauterine death occurred in 17 and 3 cases, respectively, and delivery (including premature delivery) was achieved in the remaining 180 cases. Among the 180 cases, 124 participants gave vaginal delivery, and the rest 54 cases underwent cesarean. In addition, there were 136 cases of primiparity and 64 cases of multiparity. Table 1 lists the baseline data of all participants.

Table I Baseline Data of 200 Participants

\begin{tabular}{|l|c|c|}
\hline \multicolumn{2}{|l|}{ Variables } & $\begin{array}{c}\text { Study } \\
\text { Subjects }\end{array}$ \\
\hline Age (years) & $\begin{array}{c}<0 \\
\geq 30\end{array}$ & $\begin{array}{c}27.8 \pm 3.8 \\
150(75.0 \%) \\
50(25.0 \%)\end{array}$ \\
\hline Pre-pregnancy BMI (kg/m2, mean & & $21.4 \pm 3.1$ \\
\pm SD) & & \\
\hline Fasting blood glucose (mmol/L, mean & & $4.71 \pm 0.29$ \\
\pm SD) & & \\
\hline Delivery pattern & Vaginal & $124(62.0 \%)$ \\
& Cesarean & $56(28.0 \%)$ \\
& Failed & $20(10.0 \%)$ \\
\hline Parity & Primiparity & $136(68.0 \%)$ \\
& Multiparity & $64(32.0 \%)$ \\
\hline
\end{tabular}

Abbreviation: BMI, body mass index.

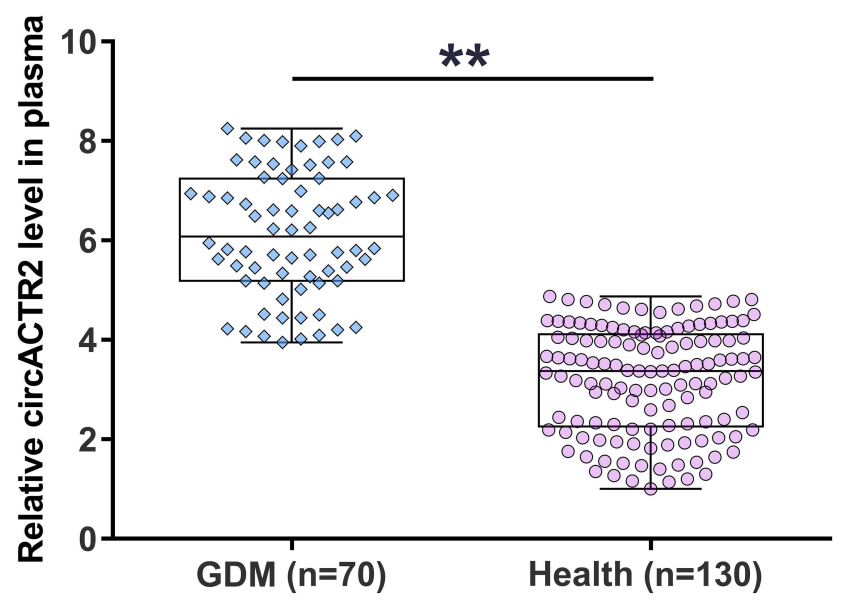

Figure I Comparison of plasma circACTR2 level between GDM and healthy groups. During the 30 weeks of follow-up, 70 pregnant women were diagnosed with GDM and were classified into the GDM group, and the rest 130 cases were grouped into the healthy group. Plasma circACTR2 levels at admission were compared between the two groups using unpaired $t$-test. $* * p<0.01$.

\section{Comparison of Plasma circACTR2 Level Between GDM and Health Groups}

During the 30 weeks of follow-up, 70 pregnant women were diagnosed with GDM and classified into the GDM group. The remaining 130 cases were grouped into the healthy group. Plasma circACTR2 levels at admission were compared between the two groups. It was observed that 70 patients presented significantly higher plasma circACTR2 levels (about 2.17-fold) than the remaining 130 patients (Figure 1, $\mathrm{p}<0.01$ ). Therefore, the increased circulating plasma circACTR2 level might correlate with the occurrence of GDM.

\section{Predictive Value of Plasma circACTR2 Level for GDM}

With the median plasma circACTR2 level at admission as the cutoff value, the 200 participants were divided into high and low circACTR2 level groups. The follow-up data were used to plot GDM-free curves for both groups. The comparison of GDM-free curves was performed with Log rank test. The high circACTR2 level group showed a higher incidence of GDM than the low circACTR2 level group (Figure 2).

\section{Association Between Plasma circACTR2 Levels and the Incidence of Adverse Events}

With the median plasma circACTR2 level of the 70 GDM patients on admission as the cutoff value, the $70 \mathrm{GDM}$ 


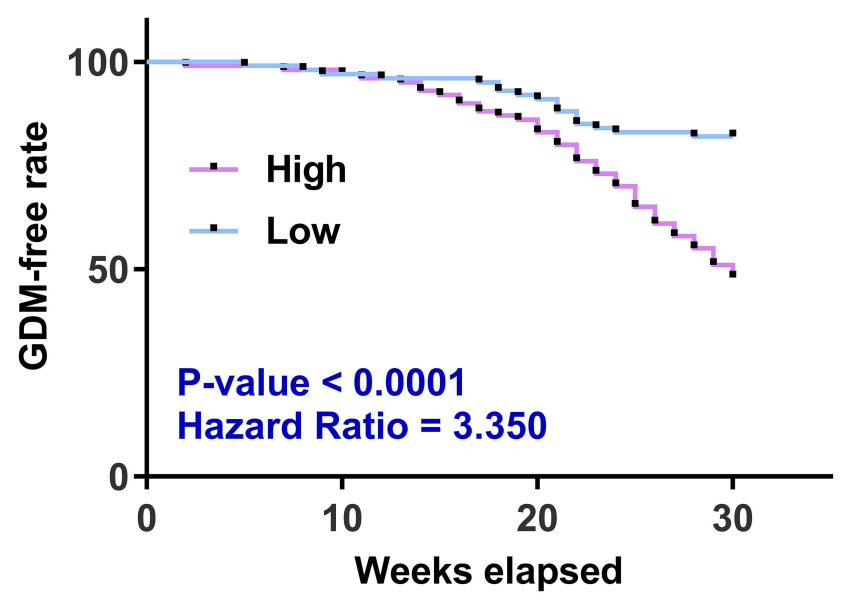

Figure 2 Analysis of the predictive value of plasma circACTR2 level for GDM. With the median plasma circACTR2 level of the 200 participants at admission as the cutoff value, the 200 participants were divided into high and low circACTR2 level groups. The follow-up data were used to plot GDM-free curves for both groups. The comparison of GDM-free free groups was performed using the Log rank test.

patients were further divided into high and low circACTR2 level groups. Associations between adverse events and plasma circACTR2 levels in these patients were analyzed by Chi-squared test or Fisher's exact test. Among the 70 patients diagnosed with GDM, high circACTR2 levels were also closely correlated with higher rates of premature delivery, miscarriage, intrauterine death, fetal malformation, intrauterine infection, and hypertension, but not macrosomia and intrauterine distress (Table 2).

\section{Discussion}

GDM is a common clinical condition caused by altered hormone secretion in the placenta in pregnant women, leading to reduced insulin utilization efficiency. This study mainly explored the involvement of circACTR2 in the occurrence of GDM and analyzed its predictive value in early GDM diagnosis and its complications.

A recent study found that circACTR2 was significantly upregulated in glucose-stressed HK-2 cells, and circACTR2 downregulation reduced IL- $1 \beta$ release, decreased pyroptosis, and attenuated productions of fibronectin and collagen IV, ${ }^{12}$ suggesting that circACTR2 could promote high glucoseinduced pyroptosis, fibrosis, and inflammation in diabetic kidney disease. ${ }^{12}$ In this study, we also observed circACTR2 upregulation in GDM, indicating its involvement in GDM. Therefore, we speculated that circACTR2 also participates in other types of diabetes and diabetic complications. ${ }^{13}$ However, the role of circACTR2 in GDM and the underlying mechanism are unclear and need to be further studied.

Differentially expressed circRNAs are frequently observed in GDM patients, and some circRNAs are potential

Table 2 Analysis of the Association Between Plasma circACTR2 Levels and the Incidence of Adverse Events Using Chi-Squared Test or Fisher's Exact Test

\begin{tabular}{|c|c|c|c|c|c|c|}
\hline Items & Groups & Cases & $\begin{array}{l}\text { High Expression } \\
\qquad(n=35)\end{array}$ & $\begin{array}{l}\text { Low Expression } \\
(n=35)\end{array}$ & $\begin{array}{c}\chi^{2} / \text { Fisher } \\
\text { Exact }\end{array}$ & p value \\
\hline Premature delivery & $\begin{array}{l}\text { Yes } \\
\text { No }\end{array}$ & $\begin{array}{l}10 \\
60\end{array}$ & $\begin{array}{c}8 \\
27\end{array}$ & $\begin{array}{c}2 \\
33\end{array}$ & 4.20 & 0.04 \\
\hline Miscarriage & $\begin{array}{l}\text { Yes } \\
\text { No }\end{array}$ & $\begin{array}{l}15 \\
55\end{array}$ & $\begin{array}{l}11 \\
24\end{array}$ & $\begin{array}{c}4 \\
31\end{array}$ & 4.16 & 0.04 \\
\hline Intrauterine death & $\begin{array}{l}\text { Yes } \\
\text { No }\end{array}$ & $\begin{array}{c}2 \\
68\end{array}$ & $\begin{array}{c}2 \\
33\end{array}$ & $\begin{array}{c}0 \\
35\end{array}$ & 0.49 & $<0.05$ \\
\hline Fetal malformation & $\begin{array}{l}\text { Yes } \\
\text { No }\end{array}$ & $\begin{array}{c}3 \\
67\end{array}$ & $\begin{array}{c}3 \\
32\end{array}$ & $\begin{array}{c}0 \\
35\end{array}$ & 0.24 & $<0.05$ \\
\hline $\begin{array}{l}\text { Intrauterine } \\
\text { infection }\end{array}$ & $\begin{array}{l}\text { Yes } \\
\text { No }\end{array}$ & $\begin{array}{c}4 \\
66\end{array}$ & $\begin{array}{c}4 \\
31\end{array}$ & $\begin{array}{c}0 \\
35\end{array}$ & 0.11 & $<0.05$ \\
\hline Hypertension & $\begin{array}{l}\text { Yes } \\
\text { No }\end{array}$ & $\begin{array}{l}20 \\
50\end{array}$ & $\begin{array}{l}16 \\
19\end{array}$ & $\begin{array}{c}4 \\
31\end{array}$ & 10.08 & 0.001 \\
\hline Macrosomia & $\begin{array}{l}\text { Yes } \\
\text { No }\end{array}$ & $\begin{array}{l}10 \\
60\end{array}$ & $\begin{array}{c}6 \\
29\end{array}$ & $\begin{array}{c}4 \\
31\end{array}$ & 0.41 & 0.49 \\
\hline Intrauterine distress & $\begin{array}{l}\text { Yes } \\
\text { No }\end{array}$ & $\begin{array}{c}2 \\
68\end{array}$ & $\begin{array}{c}1 \\
34\end{array}$ & $\begin{array}{c}1 \\
34\end{array}$ & 0.00 & 1.00 \\
\hline
\end{tabular}


early diagnostic markers for GDM. ${ }^{14}$ For instance, Yang et al showed that altered Hsa_circRNA_102893 expression could be used as an early diagnostic value for GDM. ${ }^{15}$ However, they detected Hsa_circRNA_102893 expression at 15-24 weeks of gestation, which was close to the date of GDM diagnosis. Therefore, its preventative application may not be allowed. In this study, we showed that high plasma circACTR2 level on the day of admission (about 1-month gestation) was closely correlated with high GDM occurrence during pregnancy. GDM is mostly diagnosed around 24 weeks of gestation. Therefore, analysis of circACTR2 expression could be applied as preventative approaches for patients at 1 month of gestation to prevent GDM occurrence. However, more clinical studies are needed to further analyze its accuracy.

GDM causes various adverse events in both pregnant women and infants. ${ }^{16}$ This study showed that high plasma circACTR2 level was also closely correlated with high rates of premature delivery, miscarriage, intrauterine death, fetal malformation, intrauterine infection, and hypertension, but not macrosomia and intrauterine distress. Therefore, circACTR2 may participate in certain complications of GDM and could be a target to prevent and/or treat these complications.

In this study, 70 out of 200 participants developed GDM, and the incidence is $35 \%$. Globally, the incidence of GDM ranges from $2.075 \%$ to $38.25 \% .{ }^{17}$ In China, GDM affects about $15 \%$ to $20 \%$ of pregnant women. ${ }^{18}$ We observed a much higher incidence of GDM compared to other areas of China. This is possibly because we check GDM every week, which is not practical in clinical practices. Therefore, the incidence of GDM in China might be underestimated.

\section{Conclusion}

CircACTR2 is overexpressed in GDM and predicts early GDM and multiple adverse events.

\section{Data Sharing Statement}

The data are not publicly available due to their containing information that could compromise the privacy of research participants but are available on request from the corresponding author.

\section{Ethical Approval and Consent to Participate}

All patients signed the written informed consent. All procedures were approved by the Ethics Committee of Henan
Provincial People's Hospital and operated in keeping with the standards set out in the Announcement of Helsinki and Laboratory Guidelines of Research in China.

\section{Funding}

There is no funding to report.

\section{Disclosure}

All authors declare that they do not have any commercial or associative interest that represents a conflict of interest in connection with the work submitted.

\section{References}

1. McIntyre HD, Catalano P, Zhang C, Desoye G, Mathiesen ER, Damm P. Gestational diabetes mellitus. Nat Rev Dis Primers. 2019;5(1):47.

2. Alharbi KK, Al-Sulaiman AM, Bin Shedaid MK, et al. MTNR1B genetic polymorphisms as risk factors for gestational diabetes mellitus: a case-control study in a single tertiary care center. Ann Saudi Med. 2019;39(5):309-318. doi:10.5144/0256-4947.2019.309

3. He Z, Xie H, Liang S, et al. Influence of different diagnostic criteria on gestational diabetes mellitus incidence and medical expenditures in China. J Diabetes Investig. 2019;10(5):1347-1357. doi:10.1111/ jdi.13008

4. Casagrande SS, Linder B, Cowie CC. Prevalence of gestational diabetes and subsequent Type 2 diabetes among U.S. women. Diabetes Res Clin Pract. 2018;141:200-208. doi:10.1016/j. diabres.2018.05.010

5. Li Z, Cheng Y, Wang D, et al. Incidence rate of type 2 diabetes mellitus after gestational diabetes mellitus: a systematic review and meta-analysis of 170,139 women. $J$ Diabetes Res. 2020;2020:3076463. doi:10.1155/2020/3076463

6. McKenzie-Sampson S, Paradis G, Healy-Profitós J, St-Pierre F, Auger N. Gestational diabetes and risk of cardiovascular disease up to 25 years after pregnancy: a retrospective cohort study. Acta Diabetol. 2018;55(4):315-322. doi:10.1007/s00592-017-1099-2

7. Johns EC, Denison FC, Norman JE, Reynolds RM. Gestational diabetes mellitus: mechanisms, treatment, and complications. Trends Endocrinol Metab. 2018;29(11):743-754. doi:10.1016/j. tem.2018.09.004

8. Dias S, Pheiffer C, Abrahams Y, Rheeder P, Adam S. Molecular biomarkers for gestational diabetes mellitus. Int J Mol Sci. 2018;19 (10):2926. doi:10.3390/ijms19102926

9. Wang H, She G, Zhou W, Liu K, Miao J, Yu B. Expression profile of circular RNAs in placentas of women with gestational diabetes mellitus. Endocr J. 2019;66(5):431-441. doi:10.1507/endocrj.EJ180291

10. Yan L, Feng J, Cheng F, et al. Circular RNA expression profiles in placental villi from women with gestational diabetes mellitus. Biochem Biophys Res Commun. 2018;498(4):743-750. doi:10.1016/ j.bbrc.2018.03.051

11. Zhang TN, Wang W, Huang XM, et al. Non-coding RNAs and extracellular vehicles: their role in the pathogenesis of gestational diabetes mellitus. Front Endocrinol. 2021;12:664287. doi:10.3389/ fendo.2021.664287

12. Wen S, Li S, Li L, Fan Q. circACTR2: a novel mechanism regulating high glucose-induced fibrosis in renal tubular cells via pyroptosis. Biol Pharm Bull. 2020;43(3):558-564. doi:10.1248/bpb.b19-00901

13. Desoye G, Hauguel-de Mouzon S. The human placenta in gestational diabetes mellitus. The insulin and cytokine network. Diabetes Care. 2007;30(Suppl 2):S120-S126. doi:10.2337/dc07-s203 
14. Cao M, Zhang L, Lin Y, et al. Circular RNA expression profiles in umbilical cord blood exosomes from normal and gestational diabetes mellitus patients. Biosci Rep. 2020;40:11. doi:10.1042/BSR20201946

15. Yang H, Ye W, Chen R, et al. Circulating expression of Hsa_circRNA_102893 contributes to early gestational diabetes mellitus detection. Sci Rep. 2020;10(1):19046. doi:10.1038/s41598-020-76013-5

16. Cosson E, Cussac-Pillegand C, Benbara A, et al. Pregnancy adverse outcomes related to pregravid body mass index and gestational weight gain, according to the presence or not of gestational diabetes mellitus: a retrospective observational study. Diabetes Metab. 2016;42(1):38-46. doi:10.1016/j.diabet.2015.06.001
17. Gyasi-Antwi P, Walker L, Moody C, et al. Global prevalence of gestational diabetes mellitus: a systematic review and meta-analysis. New Am J Med. 2020;1:1-10.

18. Gao C, Sun X, Lu L, et al. Prevalence of gestational diabetes mellitus in mainland China: a systematic review and meta-analysis. J Diabetes Investig. 2019;10(1):154-162. doi:10.1111/jdi.12854

\section{Publish your work in this journal}

Diabetes, Metabolic Syndrome and Obesity: Targets and Therapy is an international, peer-reviewed open-access journal committed to the rapid publication of the latest laboratory and clinical findings in the fields of diabetes, metabolic syndrome and obesity research. Original research, review, case reports, hypothesis formation, expert opinion and commentaries are all considered for publication. The manuscript management system is completely online and includes a very quick and fair peer-review system, which is all easy to use. Visit http://www.dovepress.com/testimonials.php to read real quotes from published authors. 